\title{
Black phosphorus photodetector enhanced by a planar photonic crystal cavity - Supporting Information
}

\author{
Ruijuan Tian, ${ }^{1}$ Linpeng Gu, ${ }^{1}$ Yingke Ji, ${ }^{1}$ Chen Li, ${ }^{1}$ Yuxin Chen, ${ }^{1}$ Siqi, Hu, ${ }^{1}$ Zhiwen Li, ${ }^{1}$ \\ Xuetao Gan ${ }^{1, *}$, Jianlin Zhao ${ }^{1}$ \\ 1 Key Laboratory of Light Field Manipulation and Information Acquisition, Ministry of \\ Industry and Information Technology, \\ and Shaanxi Key Laboratory of Optical Information Technology, School of Physical \\ Science and Technology, \\ Northwestern Polytechnical University, 710129 Xi' an, China \\ Corresponding Author: Email : xuetaogan@nwpu.edu.cn
}

\section{S1. Raman characterization.}

Raman spectra were acquired to determine the orientations of transferred black phosphorus (BP) flakes. ${ }^{\mathrm{S} 1, \mathrm{~S} 2}$ To facilitate the description, we define the polarization directions of the pump laser parallel and perpendicular to the carrier flow direction of BP channel with angles of $0^{\circ}$ and $90^{\circ}$, respectively, as shown in Figure S1(a).

The measured Raman spectra of the employed $3.6 \mathrm{~nm}$ and $7.9 \mathrm{~nm}$ thick BP flakes in the device fabrication are shown in Figures $\mathrm{S} 1(\mathrm{~b})$ and $\mathrm{S} 1(\mathrm{c})$. The peaks $\mathrm{A}_{\mathrm{g}}{ }_{\mathrm{g}}, \mathrm{B}_{\mathrm{g}}{ }_{\mathrm{g}}$, and $\mathrm{A}^{2} \mathrm{~g}$ are observed and the peak positions do not change as the laser polarization angle varies. This is consistent with the previous reports. ${ }^{\mathrm{S} 3}$ The spectra are normalized to the out-of-plane $\mathrm{A}_{\mathrm{g}}^{1}$ peaks since they should be independent of in-plane polarization. We can determine the orientation angles of the transferred BP flakes based on the relative ratio $r$ of the $\mathrm{B}^{2}$ g peak (zigzag direction) to the $\mathrm{A}^{2}{ }_{\mathrm{g}}$ peak (armchair direction). The $\mathrm{BP}$ crystal structure as well as the armchair and zigzag directions were also observed and demonstrated by the cross-sectional HR-STEM image in our previous work. ${ }^{\mathrm{S} 4}$

For the employed $3.6 \mathrm{~nm}$ thick BP flake of the device detailedly described in the maintext, it has $r\left(0^{\circ}\right)<r\left(90^{\circ}\right)$, which indicates the polarization direction $0^{\circ}$ is close to the armchair axis. Herein, the carrier flow direction of the BP channel is close to the armchair direction. In this case, the optical absorption by the BP flake is strong considering the transverse electric mode in the BP-integrated PPC cavity. In contrast, for the employed $7.9 \mathrm{~nm}$ thick BP flake of the device detailedly described in the 
Supporting Information, it has $r\left(0^{\circ}\right)>r\left(90^{\circ}\right)$, which indicates the polarization direction $90^{\circ}$ is close to the armchair axis. It means that the carrier flow direction in the BP channel is close to the zigzag direction. In this case, the BP absorption on the cavity mode is weak. According to the previous reports, ${ }^{\mathrm{S} 1, \mathrm{~S} 5}$ the armchair direction has the greatest optical absorption and highest carrier mobility. Therefore, the crystal orientation of the $3.6 \mathrm{~nm}$ thick BP flake is optimally aligned on the PPC cavity for maximal carrier mobility and optical absorption, while the $7.9 \mathrm{~nm}$ BP flake is not. This is also consistent with the measured high responsivity given in the main text.

(a)

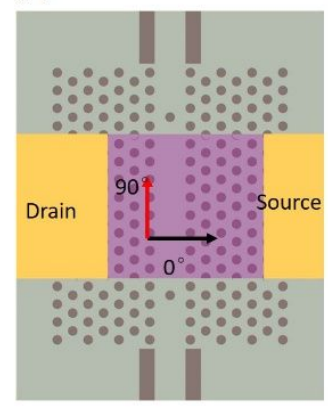

(b)

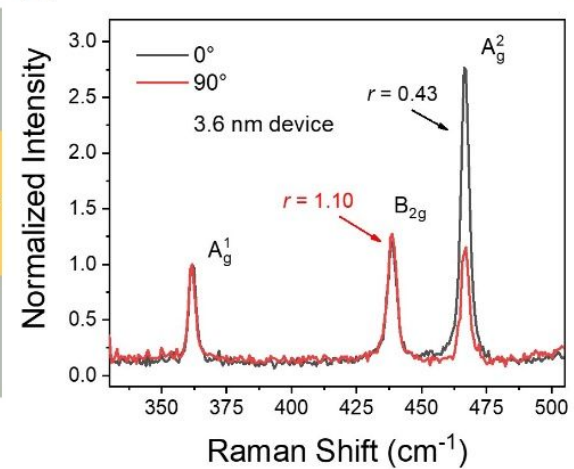

(c)

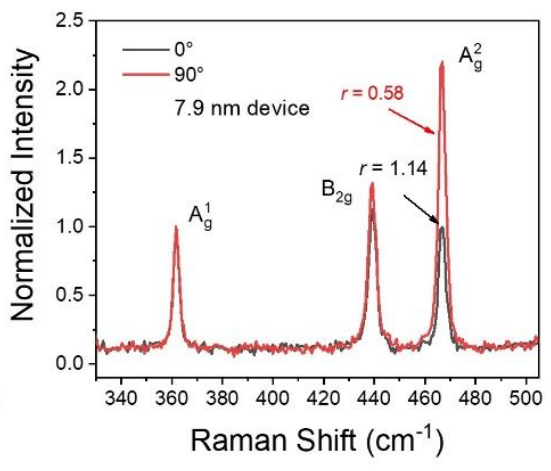

Figure S1. (a) Device structure and two polarization orientations defined for assisting the description of the Raman spectra. (b),(c) Measured Raman spectra of the $3.6 \mathrm{~nm}$ and $7.9 \mathrm{~nm}$ thick BP flakes employed in the devices described in the maintext and in the section S4 of the Supporting Information.

\section{S2. Design of the PPC cavity.}

To ensure the PPC cavity's compactness, we optimize the length of the PPC cavity. The employed PPC cavity is fabricated in a $220 \mathrm{~nm}$ thick silicon slab on a standard silicon-on-insulator (SOI) substrate with the techniques of electron beam lithography and inductively coupled plasmon etching. The PPC lattice is designed with an air-hole diameter of 240 and a lattice constant of 420 to support the photonic bandgap in the telecom-band spectral range. We first miss one line of air-holes in the PPC lattice to form a PPC waveguide. Then two air-holes are inserted in the PPC waveguide with a distance of $L$, as shown in Figure S2(a). The two air-holes function as two mirrors to confine the waveguiding mode of the PPC waveguide between them. The transmission 
spectrum of the PPC cavity could be optimized by adjusting the distance $L$ of the inserted two air-holes. In experiments, we compare the transmission spectrum for $L=$ $15 \mu \mathrm{m}$ (red line) and $L=60 \mu \mathrm{m}$ (black line), as shown in Figure S2(b). It exhibits different free spectral ranges (FSRs), which are $4.46 \mathrm{~nm}$ for $L=15 \mu \mathrm{m}$ (top panel) and $1.38 \mathrm{~nm}$ for $L=60 \mu \mathrm{m}$ (bottom panel), as the arrows indicated in Figure S2(b). The FSR is given as $\mathrm{FSR}=\Delta \lambda=\lambda^{2} / 2 n_{\mathrm{eff}} L$, where $n_{\text {neff }}$ is the effective refractive index, $L$ is the distance of the two air holes. According to this expression, it is expected to observe some strong dependence of the FSR on the length of $L$. The increase of FSRs as the reduced lengths of PPC cavity are observed. It means large FSR in the transmission spectrum could be obtained by designing a small length of the PPC cavity.

The larger FSR was adopted in this work, considering the following factors: First, as expected from the material absorption, the multiple resonance peaks in the transmission spectrum of the PPC cavity could be broadened and lowered when the BP layer was transferred on the PPC cavity. To not make the multiple resonance peaks of the transmission spectrum emergence together on the BP-integrated PPC cavity, a large FSR is needed. Second, to make the device more compact, the PPC cavity with a small length is demanded. Therefore, the $15 \mu \mathrm{m}$ length of PPC cavity is employed in this work, which has the advantage of simple design, compact footprint, large FSR and large tolerance of fabrication errors.

(a)

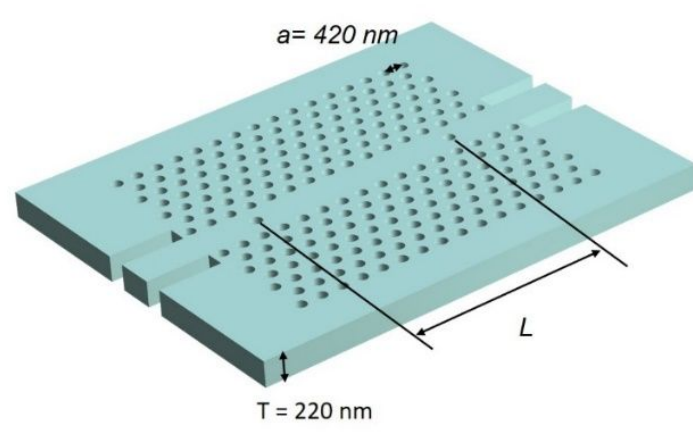

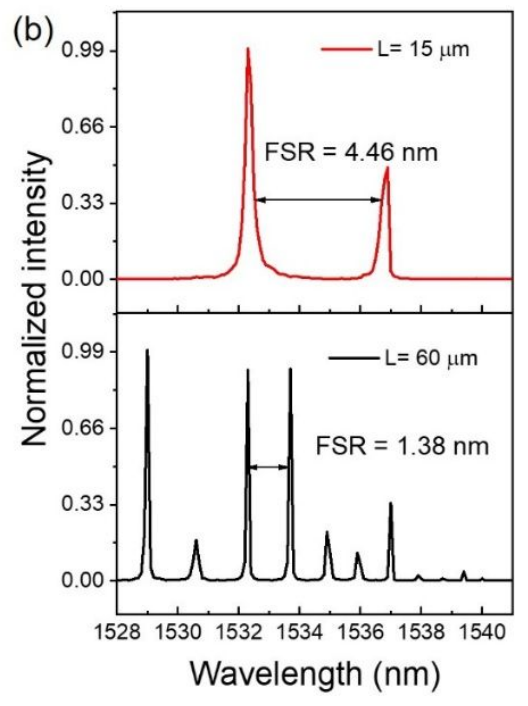


Figure S2. (a) Schematic diagram of the PPC cavity fabricated in a $220 \mathrm{~nm}$ thick silicon slab on a standard SOI substrate (b) Transmission spectra of the PPC cavities with lengths of $15 \mu \mathrm{m}$ and 60 $\mu \mathrm{m}$.

\section{S3. Model analysis for the coupled system of BP-PPC cavity.}

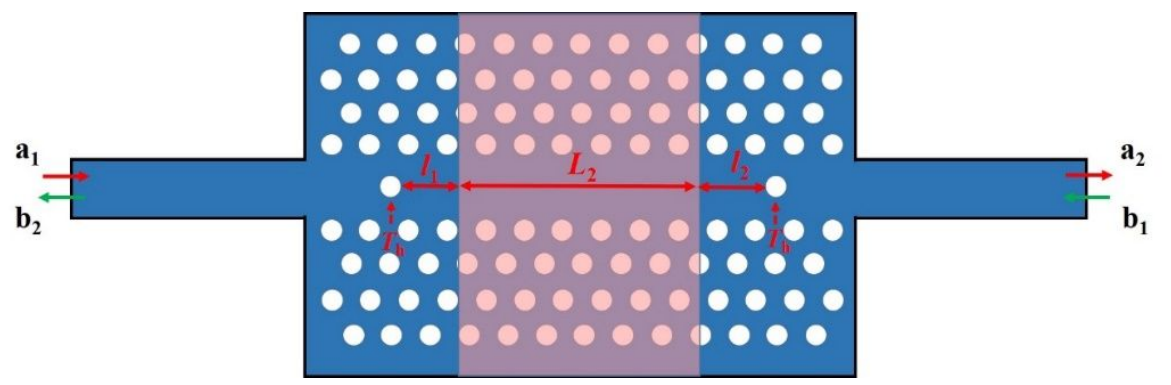

Figure S3. Schematic of the model for the coupled system of BP-PPC cavity. The PPC cavity is formed by inserting two air-holes in the PPC waveguide, which is realized by missing one line of air-holes of the PPC lattice. The bus-waveguides at two ends of the PPC waveguide have the forward (backward) propagating mode with the input and output of $a_{1}\left(b_{1}\right)$, and $a_{2}\left(b_{2}\right)$, respectively.

In this section, we describe the theoretical analysis for the coupled BP-PPC cavity system, as shown in Figure S1. Its transmission spectrum of the proposed structure could be obtained by applying a transfer matrix analysis. ${ }^{\text {S6 }}$ Here, we consider a PPC cavity with a single mode. This mode interacts with the BP and couples with the propagating modes of the waveguide. The waveguide has forward and backward propagating modes, Mode1 and Mode2. The incoming (outgoing) light of Mode1 and Mode2 are denoted by $a_{1}\left(a_{2}\right)$ and $b_{1}\left(b_{2}\right)$, respectively. The two air-holes work as partial reflectors of the propagating modes. For simplicity, they are designed with the same diameters. With an amplitude reflection coefficient of $r$, one of the air-holes generates a transfer matrix for the propagating mode of

$$
M_{h}=\frac{1}{i \sqrt{1-r^{2}}}\left[\begin{array}{cc}
-1 & -r \\
r & 1
\end{array}\right]
$$

When the light propagates through the bare waveguide section, the transfer matrices are determined by 


$$
M_{w}=\left[\begin{array}{cc}
e^{i \frac{2 \pi}{\lambda} n_{1}\left(L_{1}+L_{2}\right)} & 0 \\
0 & e^{-i \frac{2 \pi}{\lambda} n_{1}\left(L_{1}+L_{2}\right)}
\end{array}\right]
$$

Here, $n_{1}$ is the effective refractive index of the propagating mode in the bare waveguide, and $\lambda$ is the operating wavelength. The length of $L_{1}+L_{2}$ is the distance between the two air-holes in the bus waveguide, where $L_{1}=l_{1}+l_{2}$. The length of $L_{2}$ is the length of material integrated on the PPC cavity. The transfer matrices can be written as

$$
M_{w^{\prime}}=\left[\begin{array}{cc}
e^{i \frac{2 \pi}{\lambda}\left(n_{1} L_{1}+\left(n_{2}+i k\right) L_{2}\right)} & 0 \\
0 & e^{-i \frac{2 \pi}{\lambda}\left(n_{1} L_{1}+\left(n_{2}+i k\right) L_{2}\right)}
\end{array}\right]=\left[\begin{array}{cc}
a e^{i \frac{2 \pi}{\lambda}\left(n_{1} L_{1}+n_{2} L_{2}\right)} & 0 \\
0 & a^{-1} e^{-i \frac{2 \pi}{\lambda}\left(n_{1} L_{1}+n_{2} L_{2}\right)}
\end{array}\right]
$$

Where $a=\exp \left(-2 \pi k L_{2} / \lambda\right)$ is the loss from the material with the loss coefficient $k$. The transfer matrix equation for the incoming and outgoing wave amplitudes of the entire coupled system governed by

$$
\left[\begin{array}{l}
b_{1} \\
a_{2}
\end{array}\right]=M_{h} M_{w^{\prime}} M_{h}\left[\begin{array}{l}
a_{1} \\
b_{2}
\end{array}\right]
$$

To be consistent with the common operations of the experiments, we consider only the left input port has an incoming normalized light, i.e. $a_{1}=1, b_{1}=0$. The final power transmission $T(\lambda)$ and reflection $R(\lambda)$ spectrum of the coupled system could be calculated as

$$
\begin{aligned}
& T(\lambda)=\left|\frac{a_{2}}{a_{1}}\right|^{2}=\left|-\frac{t^{2}}{-r^{2} a e^{i \frac{2 \pi}{\lambda}\left(n_{1} L_{1}+n_{2} L_{2}\right)}+a^{-1} e^{-i \frac{2 \pi}{\lambda}\left(n_{1} L_{1}+n_{2} L_{2}\right)}}\right|^{2} \\
& R(\lambda)=\left|\frac{b_{2}}{a_{1}}\right|^{2}=\left|-\frac{r a e^{i \frac{2 \pi}{\lambda}\left(n_{1} L_{1}+n_{2} L_{2}\right)}-r a^{-1} e^{-i \frac{2 \pi}{\lambda}\left(n_{1} L_{1}+n_{2} L_{2}\right)}}{-r^{2} a e^{i \frac{2 \pi}{\lambda}\left(n_{1} L_{1}+n_{2} L_{2}\right)}+a^{-1} e^{-i \frac{2 \pi}{\lambda}\left(n_{1} L_{1}+n_{2} L_{2}\right)}}\right|^{2}
\end{aligned}
$$

where $t$ and $r$ are respectively the field transmission and reflection coefficients. The same expressions apply in the absence of the BP layer, with $n_{1}=n_{2}$ and $k=0$.

From Equation (S5) and (S6), we can easily deduce the BP absorption $A(\lambda)$ by the relation of $1-T-R$. We can further obtain the absorption factor $A(\lambda) / T(\lambda)$ to determine the absorption power of the BP layer, which eliminates the effect of the grating coupling 
efficiency. So the absorption power of the material is finally determined by the term of $P_{\text {absorption }}=P_{\text {transmission }} \times A(\lambda) / T(\lambda)$, in which $P_{\text {transmission }}$ is the measured transmission power through the BP-PPC cavity. The transfer matrix model to the coupled BP-PPC cavity device could be applied to other materials-resonator systems to study the interaction between electromagnetic field and material. This provides a direct relation between a measurable transmission spectrum and the absorption factor $A(\lambda) / T(\lambda)$, which does not need to consider the influence of the coupling efficiency of the whole system.

(a)

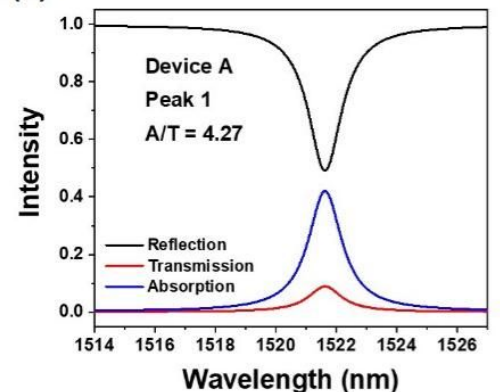

(b)

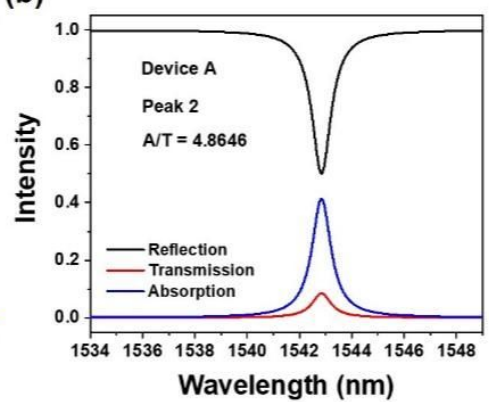

(c)

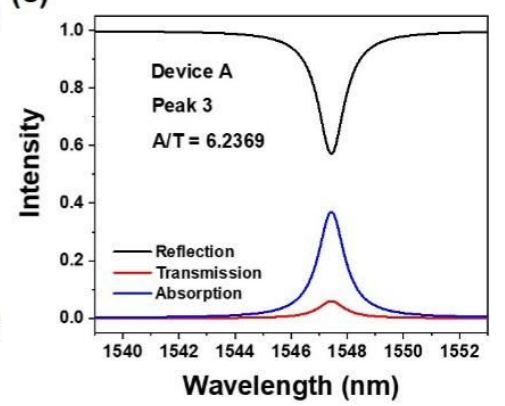

Figure S4. Calculated reflection, transmission and absorption spectrum at the three resonance wavelengths of the fabricated BP-PPC cavity shown in the maintext through transfer matrix method.

(a)

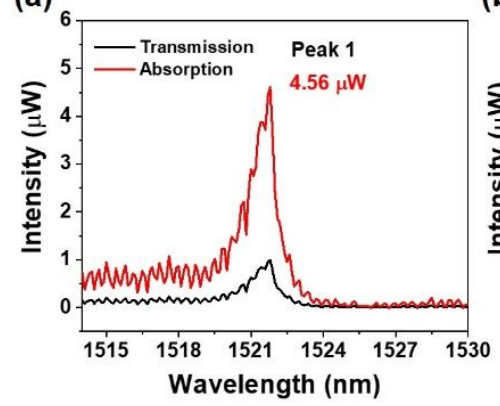

(b)

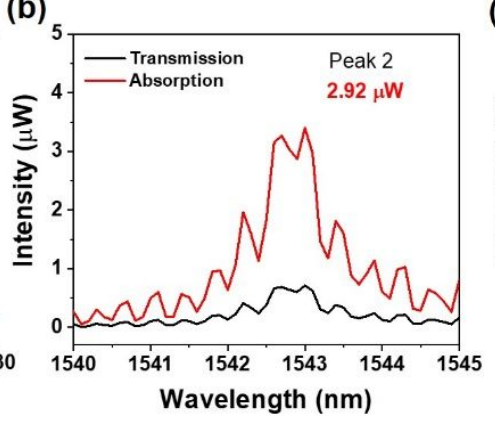

(c)

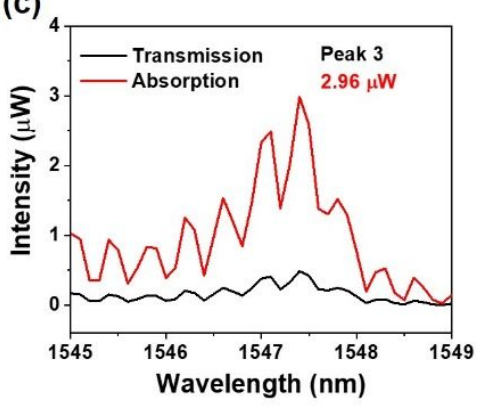

Figure S5. Measured transmission spectra and deduced absorption spectra from the device demonstrated in the maintext at the resonance wavelength of (a) $1521.65 \mathrm{~nm}$, (b) $1542.83 \mathrm{~nm}$ and (c) $1547.42 \mathrm{~nm}$.

\section{S4. Another PPC cavity-integrated BP photodetector.}

We make another device with the thicker BP flake and the gate modulation, the results are shown in Figures S6, S7, S8 and S9. This photodetector achieves a responsivity of $639 \mathrm{~mA} \mathrm{~W}^{-1}$ at the small bias of $-0.2 \mathrm{~V}$ under the gate voltage of $-2 \mathrm{~V}$ 
with a BP thickness of $7.9 \mathrm{~nm}$ (see Figures S6, S7, S8 and S9). Note that, although the photodetector with thicker BP film could achieve high responsivity and low dark current, a gate voltage must be applied to the device.

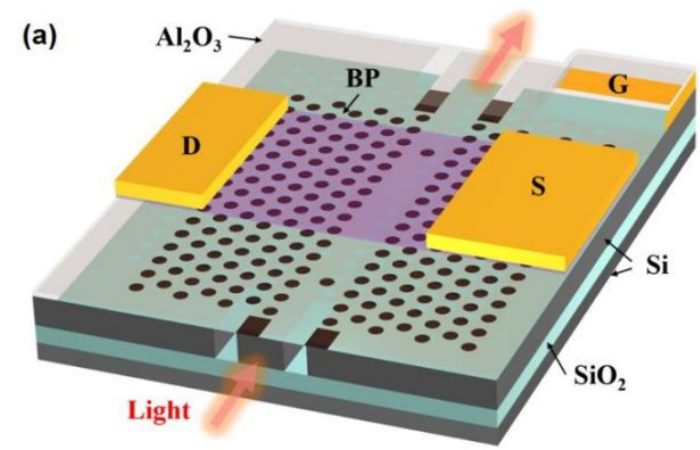

(b)

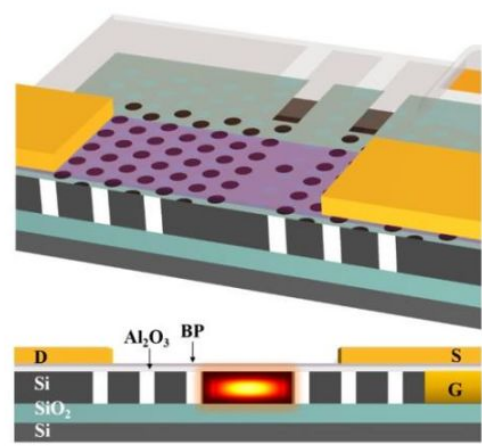

(c)

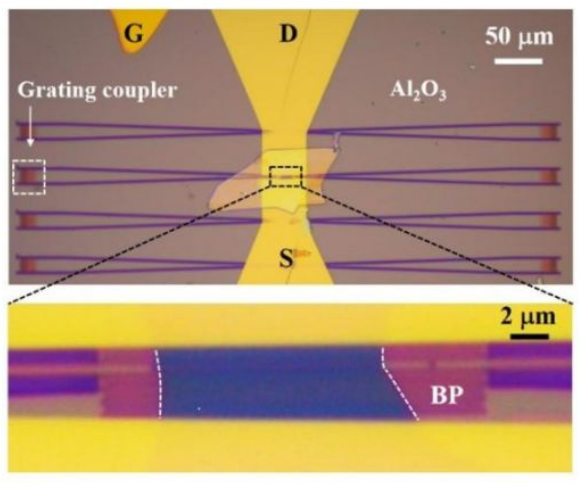

(d)

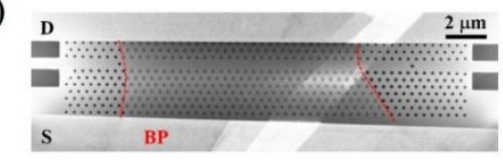

(e)

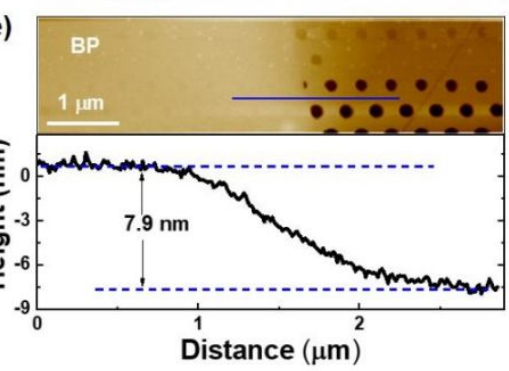

Figure S6. (a,b) Schematic of another PPC cavity-integrated BP photodetector, where D, S and G represent the drain, source and gate electrodes of the constructed BP phototransistor. (c) Top: Optical image of another BP photodetector integrated on PPC cavity. Few layer BP covers the silicon surface in the black dotted region. Two grating couplers are designed at the ends of the waveguide, as shown in the white dotted region. Two metals (source and drain) are deposited to contact BP electrically. The gate electrode directly deposited to contact the silicon. Bottom: Zoomed region of the BP flake (indicated by the white dashed lines) coating on the PPC cavity. (d) Scanning electron microscopy (SEM) image of the PPC cavity-integrated BP photodetector. (e) Top panel: Atomic force microscopy (AFM) image of the BP that integrated on the PPC cavity. Bottom panel: Height profile marked by the blue line of the top panel, indicating the thickness of the BP flake is $7.9 \mathrm{~nm}$. 
(a)

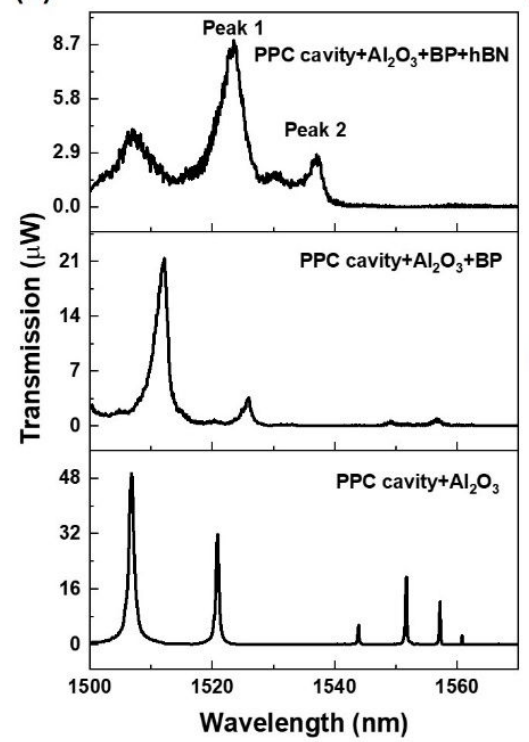

(b)
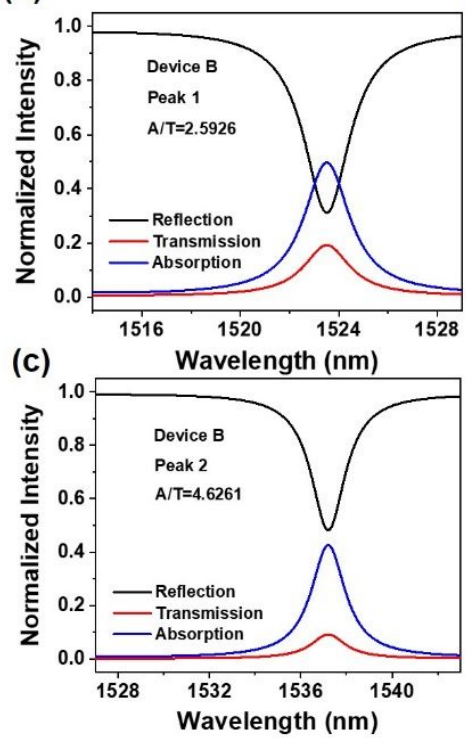

(d)

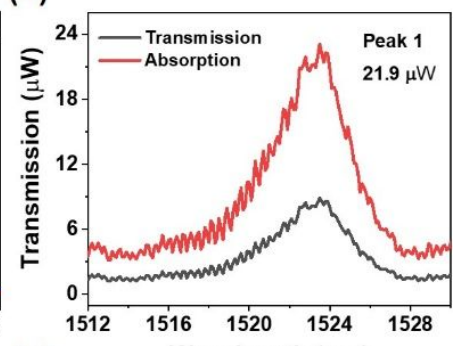

(e)

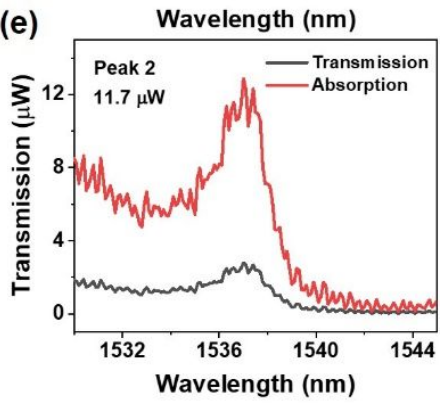

Figure S7. (a) Transmission spectra of the PPC cavity measured in each fabrication process for the device with the BP thickness of $7.9 \mathrm{~nm}$. (b), (c) Calculated reflection, transmission and absorption spectra at the two resonance wavelengths using transfer matrix method in the device with the BP thickness of $7.9 \mathrm{~nm}$. (d), (e) Transmission spectra measured in experiments (black curves) and deduced absorption spectra (red curves) from the extracted factor $A(\lambda) / T(\lambda)$ in the transfer matrix model at the two resonance wavelengths for the device.

(a)

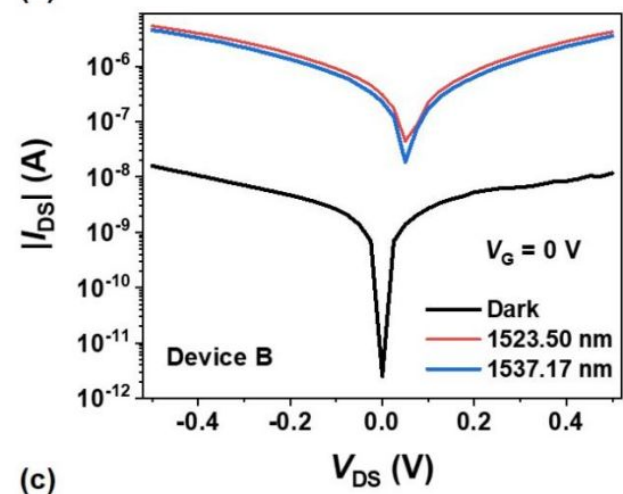

(c)

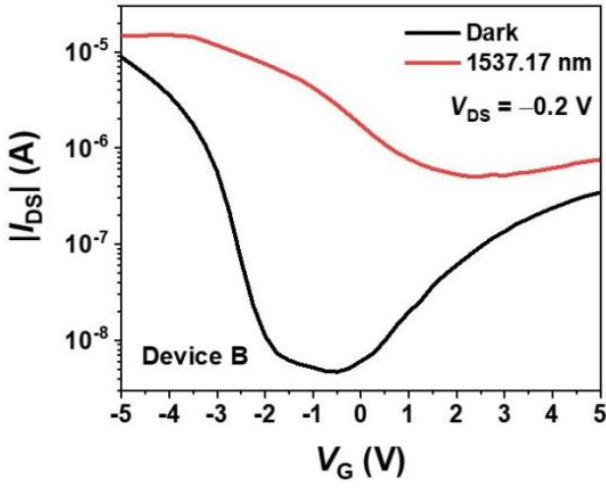

(b)

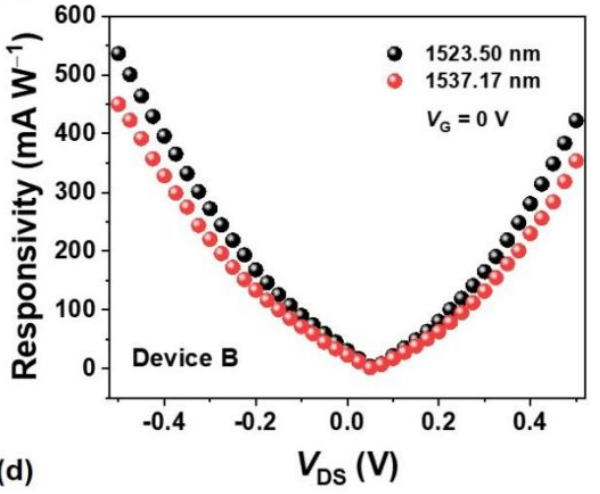

(d)

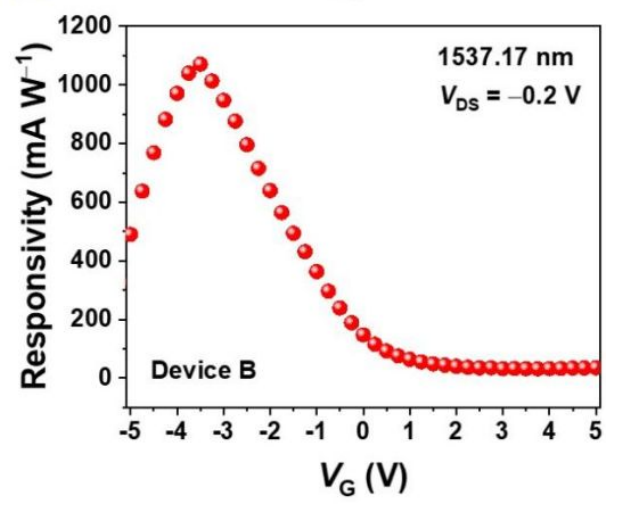


Figure S8. Photoresponse of the device with the BP thickness of $7.9 \mathrm{~nm}$ by controlling the bias and gate voltages. (a) Measured $I_{\mathrm{DS}}-V_{\mathrm{DS}}$ characteristics of the photodetector without light illumination and with light incidence at two resonance wavelengths. (b) Responsivity as the function of the bias voltage at zero gate voltage $V_{\mathrm{G}}=0 \mathrm{~V}$ at the two resonance peaks. (c) Transfer curve under the bias of $-0.5 \mathrm{~V}$ at dark and under the light illumination at a wavelength of $1523.50 \mathrm{~nm}$. (d) Responsivity as the function of the gate voltage at the bias voltage of $V_{\mathrm{DS}}=-0.2 \mathrm{~V}$.

(a)

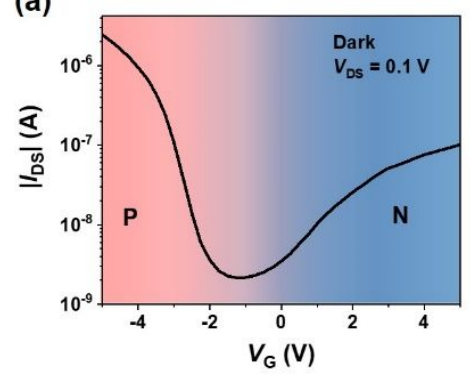

(b)

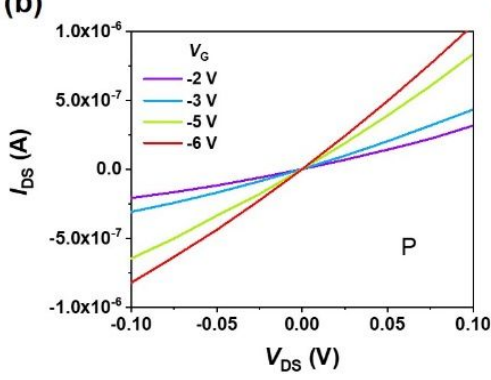

(c)

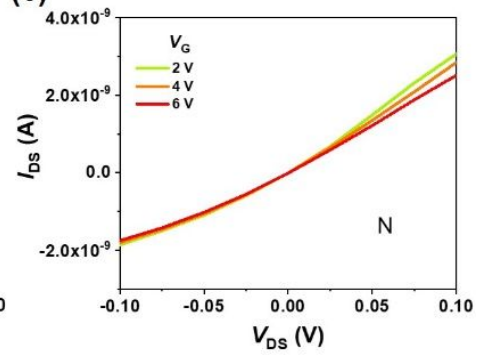

Figure S9. (a) Transfer characteristic at $V_{\mathrm{DS}}=0.1 \mathrm{~V}$ in the device with the BP thickness of $7.9 \mathrm{~nm}$.

(b) Output characteristics for $V_{\mathrm{G}}$ from -2 to $-6 \mathrm{~V}$ in the device with the BP thickness of $7.9 \mathrm{~nm}$. (c)

Output characteristics for $V_{\mathrm{G}}$ from 2 to $6 \mathrm{~V}$ in the device with the BP thickness of $7.9 \mathrm{~nm}$.

S5. Impulse response measurement for the device.

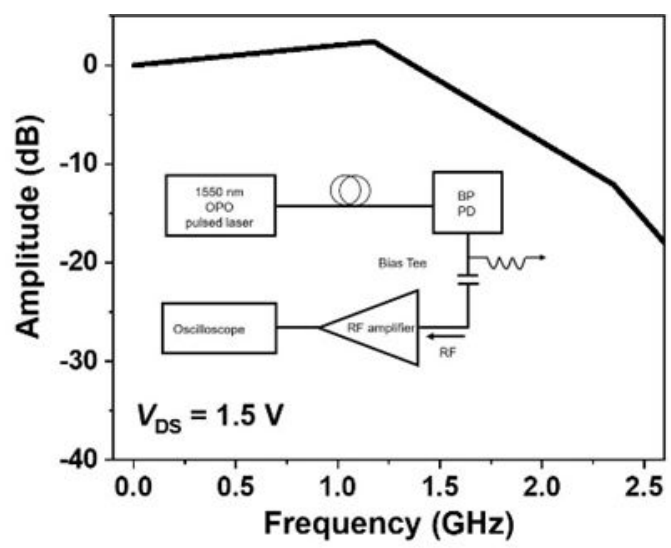

Figure S10. Fourier transformation of the impulse response for the BP photodetector in the maintext. Inset is the measurement setup of the impulse response. A $3 \mathrm{~dB}$ bandwidth of $\sim 1.42 \mathrm{GHz}$ is obtained.

Figure S10 plots the Fourier transformation of the pulse for the BP photodetector in the maintext, which yields the same bandwidth. The inset is the impulse measurement setup. The operation speed of $\sim 1.4 \mathrm{GHz}$ obtained in our device is limited by our 
measurement instrument. The employed RF current amplifier (Mini-circuits, ZKL1R5+, 10-1500 MHz) has a upper limit response bandwidth around 1.5 GHz. We also analyze the RC constant and transit time of our device, which are normally considered as two of the most important factors limiting response speed of photodetectors.

The $R C$-time of our device is analyzed as follows: According to the currentvoltage curve measured on the photodetector by the RF GS probe, a resistance $R$ of $\sim 9$ $\mathrm{k} \Omega$ is derived. Because of the absence of instruments to measure the exact capacitance of our device, we estimated it by referring to the values reported in another work about BP photodetector. ${ }^{\mathrm{S} 2}$ Considering the channel length and width as well as the dimensions of the electrodes of our deivces are close to that employed in Ref. S2, we estimated the capacitance $C$ of our device is around $5 \mathrm{fF}$. The $3 \mathrm{~dB}$ response bandwidth $f_{3 \mathrm{~dB}} \approx 1 / 2 \pi R C$ $\approx 3.5 \mathrm{GHz}$, which is larger than the measured $1.42 \mathrm{GHz}$.

For the transit time of our fabricated device, it is calculated by $\tau_{\mathrm{tr}}=l^{2} /\left(2 \mu V_{\text {bias }}\right)$, where the BP channel length $l$ is $3.4 \mu \mathrm{m}$, the $V_{\text {bias }}$ is set to be $1.5 \mathrm{~V}$. The carrier mobility is estimated around $\sim 10^{3}\left(\mathrm{~cm}^{2} / \mathrm{V} \cdot \mathrm{s}\right)$ according to the previously reported values. ${ }^{\mathrm{S} 1, \mathrm{~S} 7}$ So the transit time is calculated about 38.5 ps. Based on the extracted parameters of the saturation time $\tau_{\mathrm{s}}(64 \mathrm{ps})$ and the recombination time $\tau_{\mathrm{r}}(240 \mathrm{ps})$ from the intrinsic BP, 8 the response time $\tau$ is about 71.8 ps. The corresponding $3 \mathrm{~dB}$ response bandwidth around $f_{3 \mathrm{~dB}}=0.44 / \tau \approx 6.1 \mathrm{GHz}$, which is larger than the $1.42 \mathrm{GHz}$ measured from our devices as well.

\section{S6. Comparison with prior-art 2D material photodetectors integrated on silicon waveguides or microcavities.}

The following table serves as a comparison with the current state-of-the-art 2D material photodetectors integrated on silicon waveguides and microcavities.

Table S1: Comparison of our work with other 2D material photodetectors integrated on silicon waveguides or microcavities.

\begin{tabular}{|c|c|c|c|c|c|c|}
\hline $\begin{array}{c}\text { Integrated } \\
\text { Photonic }\end{array}$ & $\begin{array}{c}\text { Waveleng } \\
\text { th }\end{array}$ & $\begin{array}{c}\text { 2D } \\
\text { material }\end{array}$ & $\mathbf{I}_{\text {dark }}$ & Responsivity & $\begin{array}{c}\text { Response } \\
\text { speed }\end{array}$ & Ref. \\
\hline
\end{tabular}




\begin{tabular}{|c|c|c|c|c|c|c|}
\hline structures & & & & & & \\
\hline \multirow{5}{*}{ Waveguide } & $1160 \mathrm{~nm}$ & $\mathrm{MoTe}_{2}$ & $10^{-6}$ & $5 \mathrm{~mA} / \mathrm{W}(2 \mathrm{~L})$ & $200 \mathrm{MHz}$ & $\mathrm{S} 9$ \\
\cline { 2 - 7 } & $1310 \mathrm{~nm}$ & $\mathrm{MoTe}_{2}$ & $2 \times 10^{-8}$ & $\begin{array}{c}23 \mathrm{~mA} / \mathrm{W}(19.5 \mathrm{~nm}) \\
400 \mathrm{~mA} / \mathrm{W}(60 \mathrm{~nm})\end{array}$ & $500 \mathrm{MHz}$ & $\mathrm{S} 10$ \\
\cline { 2 - 7 } & $1550 \mathrm{~nm}$ & $\mathrm{BP}$ & $2.2 \times 10^{-7}$ & $\begin{array}{c}135 \mathrm{~mA} / \mathrm{W}(11.5 \mathrm{~nm}) \\
657 \mathrm{~mA} / \mathrm{W}(100 \mathrm{~nm})\end{array}$ & $3 \mathrm{GHz}$ & $\mathrm{S} 2$ \\
& $1550 \mathrm{~nm}$ & Graphene & $5 \times 10^{-5}$ & $76 \mathrm{~mA} / \mathrm{W}$ & $65 \mathrm{GHz}$ & $\mathrm{S} 11$ \\
\hline \multirow{5}{*}{ Microcavity } & $850 \mathrm{~nm}$ & Graphene & - & $21 \mathrm{~mA} / \mathrm{W}$ & - & $\mathrm{S} 12$ \\
\cline { 2 - 7 } & $1500-$ & Graphene & - & $0.6 \mathrm{~mA} / \mathrm{W}$ & - & $\mathrm{S} 13$ \\
\cline { 2 - 7 } & $1550 \mathrm{~nm}$ & & & & & \\
\cline { 2 - 7 } & $1500-$ & BP & $2 \times 10^{-8}$ & $125 \mathrm{~mA} / \mathrm{W}(3.6 \mathrm{~nm})$ & $1.42 \mathrm{GHz}$ & This \\
& $1550 \mathrm{~nm}$ & & $1.1 \times 10^{-8}$ & $639 \mathrm{~mA} / \mathrm{W}(7.9 \mathrm{~nm})$ & & work \\
\hline
\end{tabular}

We compare the performance of our silicon PPC cavity-integrated BP photodetector with other reported 2D material photodetectors integrated on waveguides or microcavities, as shown in Table S1. All of these 2D material photodetectors were proposed to provide chip-integrated photodetectors for silicon photonics. Limited by the moderately large bandgaps of the few-layer $\mathrm{MoTe}_{2}$, it is a challenge to fabricate $\mathrm{MoTe}_{2}$ based photodetectors operating at the telecom-band around $1550 \mathrm{~nm}$. On contrary, due to the zero bandgap of graphene, and narrow bandgap of BP, their photodetectors could response light around the wavelength range of $1550 \mathrm{~nm}$ for silicon photonics. Unfortunately, the zero bandgap of graphene causes large dark current in its photodetector. Compared with the BP photodetectors integrated on the silicon waveguide, our BP photodetector integrated on the silicon PPC cavity has smaller dark current and higher internal quantum efficiency. This could be attributed to the cavityenhanced light-matter interaction on the BP flake, which improves the light absorption as well as the photoresponse. Also, the cavity-integration architecture has much smaller device footprint than the waveguide-integration, which therefore could reduce the dark current and promise much higher response speed, as illustrated in the maintext. Here, the measured $1.42 \mathrm{GHz}$ response speed is limited by the measurement instruments. In addition, our PPC cavity-integrated BP photodetector achieves a 36-fold enhancement of photocurrent at the telecom-band, which is the highest enhancement factor realized in the reported cavity-enhanced 2D materials photodetectors. 
Reference

(S1) Xia, F.; Wang, H.; Jia, Y. Rediscovering Black Phosphorus as an Anisotropic Layered Material for Optoelectronics and Electronics. Nat. Commun. 2014, 5, 4458.

(S2) Youngblood, N.; Chen, C.; Koester, S. J.; Li, M. Waveguide-Integrated Black Phosphorus Photodetector with High Responsivity and Low Dark Current. Nat. Photonics 2015, 9 (4), 247-252.

(S3) Xu, M.; Gu, Y.; Peng, R.; Youngblood, N.; Li, M. Black Phosphorus MidInfrared Photodetectors. Appl. Phys. B 2017, 123 (4), 130.

(S4) Tian, R.; Fei, R.; Hu, S.; Li, T.; Zheng, B.; Shi, Y.; Zhao, J.; Zhang, L.; Gan, X.; Wang, X. Observation of Excitonic Series in Mono- and Few-Layer Black Phosphorus. Phys. Rev. B 2020, 101, 235407.

(S5) Low, T.; Engel, M.; Steiner, M.; Avouris, P. Origin of Photoresponse in Black Phosphorus Phototransistors. Phys. Rev. B 2014, 90 (8), 081408(R).

(S6) Fan, S. Sharp Asymmetric Line Shapes in Side-Coupled Waveguide-Cavity Systems. Appl. Phys. Lett. 2002, 80 (6), 908-910.

(S7) Qiao, J.; Kong, X.; Hu, Z. X.; Yang, F.; Ji, W. High-Mobility Transport Anisotropy and Linear Dichroism in Few-Layer Black Phosphorus. Nat. Commun. 2014, 5, 4475.

(S8) Youngblood, N.; Li, M. Ultrafast Photocurrent Measurements of a Black Phosphorus Photodetector. Appl. Phys. Lett. 2017, 110 (5), 051102.

(S9) Bie, Y.-Q.; Grosso, G.; Heuck, M.; Furchi, M. M.; Cao, Y.; Zheng, J.; Bunandar, D.; Navarro-Moratalla, E.; Zhou, L.; Efetov, D. K.; Taniguchi, T.; Watanabe, K.; Kong, J.; Englund, D.; Jarillo-Herrero, P. A MoTe 2 -Based Light-Emitting Diode and Photodetector for Silicon Photonic Integrated Circuits. Nat. Nanotechnol. 2017, 12 (12), 1124-1129.

(S10) Ma, P.; Flöry, N.; Salamin, Y.; Baeuerle, B.; Emboras, A.; Josten, A.; Taniguchi, T.; Watanabe, K.; Novotny, L.; Leuthold, J. Fast $\mathrm{MoTe}_{2}$ Waveguide Photodetector with High Sensitivity at Telecommunication Wavelengths. ACS Photonics 2018, 5 (5), 1846-1852. 
(S11) Schuler, S.; Schall, D.; Neumaier, D.; Dobusch, L.; Bethge, O.; Schwarz, B.; Krall, M.; Mueller, T. Controlled Generation of a P-n Junction in a Waveguide Integrated Graphene Photodetector. Nano Lett. 2016, 16 (11), 7107-7112.

(S12) Furchi, M.; Urich, A.; Pospischil, A.; Lilley, G.; Unterrainer, K.; Detz, H.; Klang, P.; Andrews, A. M.; Schrenk, W.; Strasser, G.; Mueller, T. MicrocavityIntegrated Graphene Photodetector. Nano Lett. 2012, 12 (6), 2773-2777.

(S13) Shiue, R. J.; Gan, X.; Gao, Y.; Li, L.; Yao, X.; Szep, A.; Walker, D.; Hone, J.; Englund, D. Enhanced Photodetection in Graphene-Integrated Photonic Crystal Cavity. Appl. Phys. Lett. 2013, 103 (24), 241109. 